\title{
Auraptene Inhibits Migration and Invasion of Cervical and Ovarian Cancer Cells by Repression of Matrix Metalloproteinasas 2 and 9 Activity
}

\author{
Khadijeh Jamialahmadi ${ }^{1,2}$, Sofia Salari ${ }^{4}$, Nafiseh Sadat Alamolhodaei ${ }^{3}$, Amir Avan $^{5}$, \\ Leila Gholami', Gholamreza Karimi ${ }^{4,7 *}$
}

\begin{abstract}
'Biotechnology Research Center, Pharmaceutical Technology Institute, Mashhad University of Medical Sciences, Mashhad, Iran ${ }^{2}$ Department of Medical Biotechnology, Faculty of Medicine, Mashhad University of Medical Sciences, Mashhad, Iran

${ }^{3}$ Biotechnology Research Center, School of Pharmacy, Mashhad University of Medical Sciences, Mashhad, Iran

${ }^{4}$ Pharmaceutical Research Center, Institute of Pharmaceutical Technology, Mashhad University of Medical Sciences, Mashhad, Iran

${ }^{5}$ Metabolic Syndrome Research Center, Mashhad University of Medical Sciences, Mashhad, Iran

${ }^{6}$ Department of Modern Sciences and Technologies, School of Medicine, Mashhad University of Medical Sciences, Mashhad, Iran

${ }^{7}$ Department of Pharmacodynamics and Toxicology, School of Pharmacy, Mashhad University of Medical Sciences, Mashhad, Iran
\end{abstract}

\section{Key Words}

auraptene, cancer, migration, invasion, matrix metalloproteinases

\section{Abstract}

Objectives: Auraptene, a natural citrus coumarin, found in plants of Rutaceae and Apiaceae families. In this study, we investigated the effects of auraptene on tumor migration, invasion and matrix metalloproteinase (MMP)-2 and -9 enzymes activity.

Methods: The effects of auraptene on the viability of A2780 and Hela cell lines was evaluated by MTT assay. Wound healing migration assay and Boyden chamber assay were determined the effect of auraptene on migration and cell invasion, respectively. MMP-2 and MMP-9 activities were analyzed by gelatin zymography assay.

Results: Auraptene reduced A2780 cell viability. The results showed that auraptene inhibited in vitro migration and invasion of both cells. Furthermore, cell invasion ability suppressed at $100 \mu \mathrm{M}$ auraptene in Hela cells and at 25, 50 $\mathrm{M}$ in A2780 cell line. Gelatin zymography

Received: Apr 04, 2018 Reviewed: Jun 20, 2018 Accepted: Aug 02, 2018

$@$ This is an Open-Access article distributed under the terms of the Creative Common Attribution Non-Commercial License (http://creativecommons.org/licenses/by-nc/4.0/) which permits unrestricted noncommercial use, distribution, and reproduction in any medium, provided the original work is properly cited.

(2) This paper meets the requirements of KS X ISO 9706, ISO 9706-1994 and ANSI/NISO Z39.48-1992 (Permanence of Paper). showed that for Hela cell line, auraptene suppressed MMP-2 enzymatic activity in all concentrations and for MMP-9 at a concentration between 12.5 to $100 \mu \mathrm{M}$ in A2780 cell line.

Conclusion: Auraptene inhibited migration and invasion of human cervical and ovarian cancer cells in vitro by possibly inhibitory effects on MMP-2 and MMP-9 activity.

\section{Introduction}

Cervical and ovarian cancers are the fourth and the fifth common cancers among women worldwide, respectively. Interestingly, more than $85 \%$ of all cervical cancers and its mortality occur in developing countries $[1,2]$. In ovarian cancer, the average time of clinical remission is approximately 2 years. Hence, development in cancer therapy process seems quite necessary [3]. Although these female cancers are treatable in early stages, many cases are diagnosed at an advanced stage after metastasis has occurred so resulting in a poor prognosis and treatment failure.

Matrix metalloproteinases (MMPs) are a large group of zinc-dependent endopeptidases which are responsible for extracellular matrix (ECM) dissociation. MMPs have an important role in angiogenesis $[4,5]$, tumor

E-mail: KarimiG@mums.ac.ir 
proliferation and migration or metastasis [6, 7]. Among the various MMPs, MMP-2 (gelatinase A) and MMP-9 (gelatinase B) are closely related to tumor invasion and matastasis [8]. As a rule, ECM dissociation by MMPs is one way to metastasis; Therefore identification novel chemotherapeutics for the matrix metalloproteinase inhibition may be a promising strategy for blocking migration and tumor metastasis [9].

Auraptene is a well-known oxi-coumarin was first extracted from, Citrus aurantium, which is one of the citrus species $[10,11]$.

Previous studies have shown that auraptene has various valuable properties including anti-inflammatory [12], anti-oxidant [13], anti-coagulant [14], anti-microbial [15], anti-cancer, neuroprotective [16], and immuno-modulatory effects $[14,17,18]$.

Krishnan et al demonstrated that auraptene reduced cell proliferation at concentration of 20-50 $\mu \mathrm{M}$ in MCF-7 breast cancer cells [19].

In another study, auraptene decreased the incidence of colon adenocarcinoma at $100,500 \mu \mathrm{g} / \mathrm{ml}$ during the initiation and post initiation. Also, auraptene inhibited the development of azoxymethane (AOM)-induced precursor lesions for colorectal carcinoma [20].

Furthermore, a recent study showed that auraptene could suppress the Dextran sulfate sodium (DSS)-induced gelatinolytic activity of MMP-7 as well as the expression of MMP-2 and MMP-9 in ulcerative colitis in mice. Although the anticancer effects of auraptene in some cancer cells has been shown, its involvement in growth inhibition and lowering of cervical and ovarian cancer cells invasion remain unknown. In this study, we aimed to investigate the effect of auraptene as notable citrus coumarin on the growth capacity of two cancer cell lines, Hela and A2780 as cervical and ovarian cancers, respectively. Additionally, due to the pivotal role of MMP-2 and MMP-9 in tumor proliferation, migration and invasion, the inhibitory effect of auraptene on MMPs activity was reported [21].

\section{Material and Methods}

\subsection{Cell lines and Reagents}

A2780 and Hela cell lines from Pasture Institute (Tehran, Iran); Dimethyl Sulfoxide (DMSO), Triton X-100, penicillin / streptomycin, Sodium dodecyl sulphate, Tris- $\mathrm{HCl}$ and Giemsa from Sigma (Saint Louis, MO, USA); RPMI-1640 , FBS and phosphate-buffered saline from Gibco; 3-(4,5-Dimethylthiazol-2-yl)-2,5-Diphenyltetrazolium Bromide (MTT) from Milipore (USA); auraptene from Dr. Iranshahi, Iran; Tetramethyl ethylene diamine, Bromophenol Blue and Kumasi Blue R-250 from Merck (Germany).

\subsection{Cell culture and treatments}

A2780 and Hela cell lines were cultured inRPMI-1640 supplemented with $10 \%$ fetal bovine serum and $100 \mathrm{u} / \mathrm{ml}$ penicillin and $100 \mu \mathrm{g} / \mathrm{ml}$ streptomycin at $37^{\circ} \mathrm{C}$ in the presence of $5 \% \mathrm{CO} 2$. After preparation of auraptene stock $(10 \mathrm{mM})$ (preparation with DMSO), the concentration of auraptene at $(0.78125,1.5625,3.125,6.25,12.5,25,50100 \mu \mathrm{M})$ were provided by dilution in cell culture medium [3].

\subsection{Cytotoxicity assay}

The cytotoxicity of auraptene on two cell lines (A2780 and Hela) was evaluated after cell counting and seeding 104 cells in 96 well plates. After overnight incubation, the medium was removed and $100 \mu \mathrm{l}$ media supplemented with increasing concentrations of auraptene $(0.78125,1.5625$, $3.125,6.25,12.5,25,50,100 \mu \mathrm{M}$ ) were added to wells with five repetitions.

After 24 hours incubation with auraptene and the medium removal, cells were stained by $20 \mu \mathrm{L}$ MTT solutions and were incubated for 3 hours. The medium in each well was carefully removed, and then $100 \mu \mathrm{L}$ of DMSO were added to each well. Finally, the absorption of solubilized formazanin each well was measured at $570 \mathrm{~nm}$. Values were corrected for background absorbance at $630 \mathrm{~nm}$ [3].

\subsection{Wound healing migration assay}

The wound healing migration assay is the study of cell migration and cell interactions. First a straight scratch is made (simulating a wound) and then cell ability to wound healing is detected [22], related to time and auraptene with different concentrations. In this experiment, both cell lines ( $5 \times 104$ cells) were cultured in 24 well plates. After culture confluences, the monolayer cells were scratched, using a sterile (yellow) pipette tip to create a denuded zone (gap) of constant width. Each well was washed with PBS to remove the cellular debris and then exposed to different concentrations of auraptene $(6.25,12.5,25,50,100 \mu \mathrm{M})$. Both cell lines incubated at $37^{\circ} \mathrm{C}$ in the presence of $5 \%$ $\mathrm{CO} 2$. Hela cells migrated to the wounded region was photographed at 0, 6, 24 hours for A2780 cells at 0, 24, 48, 72 hours. Finally, the wound area was measured by the program Image J software [23].

\subsection{Matrigel invasion assay}

In order to study the effect of auraptene on the cell invasive capacity, a modified Boyden-chamber technique with matrigel-coated membranes was used. Briefly, 105 from each cell line was placed onto the upper chamber and treated with auraptene at concentrations of $(6.25,12.5,25$, $50,100 \mu \mathrm{M})$ and $(6.25,12.5,25,50 \mu \mathrm{M})$ for Hela and A2780 cells, respectively. The complete medium (containing 10\% FBS) was applied to the lower chamber as chemoattractant. After overnight incubation, the cells in the upper surface of the membrane were carefully removed with a cotton swab.

Finally, the cells that had penetrated the matrigel membrane and invaded to the lower surface of the membrane, were fixed with methanol and stained with $10 \%$ Gimsa color solution for $15 \mathrm{~min}$ and counted under the microscope [24]. 


\subsection{Gelatin zymography}

To measure enzymatic activity of MMP-2 and 9 as the key enzymes in the cell invasion and metastasis, gelatin zymography were examined. Cells $(5 \times 105$ cells $/ \mathrm{ml})$ were seeded in 6-well plates. After overnight incubation, Hela cells were treated with auraptene at concentrations of $(6.25,12.5,25,50,100 \mu \mathrm{M})$ and $\mathrm{A} 2780$ cells at concentration of $(6.25,12.5,25,50 \mu \mathrm{M})$. At the end of incubation periods, upper medium was centrifuged at $400 \mathrm{~g}$ for $5 \mathrm{~min}$. Then, $12 \mu \mathrm{l}$ of supernatant aliquots of culture medium from each concentration was mixed with $3 \mu$ l of loading buffer $5 x$. Samples were then loaded on to $8 \%$ acrylamide gels containing $0.1 \%$ gelatin as a substrate and the electrophoresis was done at $110 \mathrm{v}$ for 2 hours.

For revitalizing structure and activity of the MMP enzymes, gels were inserted into developing buffer at $37^{\circ} \mathrm{C}$ for 42 hours and then stained with Coomasie brilliant blue R-250 for 1 hour at room temperature.

After decolorization and gel photographing, Image J software was used for counting the color density at band formation area [25].

\subsection{Statistical analysis}

Each experiment was repeated three to four times. Data were expressed as mean \pm standard deviation and analyzed by one-way analyses of variance with ANOVA and Dunnett's posttest. Differences were considered statistically significant when $\mathrm{p}$-values were $<0.05$.

\section{Results}

\subsection{The effects of auraptene on Hela and $\mathbf{A} 2780$ cells viability}

As shown in (Fig.1), cell viability was examined by MTT assay. Exposure to auraptene for 24 hours significantly reduced cell viability at concentration of $25-100 \mu \mathrm{M}$ in Hela cells (IC50: $47.93 \mu \mathrm{M}$ ) and 6.25-100 $\mu \mathrm{M}$ in A2780 cells (IC50:31.49 $\mu \mathrm{M})$. Moreover, maximum toxicity was observed at concentration of $100 \mu \mathrm{M}$ in both cell lines.

\subsection{Inhibitory effect of auraptene on Hela cell migration}

The anti-migratory effects of auraptene on cervical and ovarian cancer cells was evaluated by Wound healing migration assay. In Hela cell line, significant change in cell migration was found at 50,100 $\mathrm{M}$ auraptene after 6 hours of incubation and at $100 \mu \mathrm{M}$ after 24 hours, compared to control. Also, the percent of migration was $100 \%$ in other concentrations, after 24 hours (Fig.2B).

\subsection{Inhibitory effect of auraptene on A2780 cells migration}

After incubation with various concentrations of aurap- tene for 48 hours, auraptene significantly inhibit the cell migration at $12.5,25,50 \mu \mathrm{M}$. Also, after 72 hours incubation, cell migration significantly decreased in all concentrations (Fig.3B).

\subsection{Inhibitory effect of auraptene on cell invasion}

The inhibitory effect of auraptene on the invasion of Hela and A2780 cells were demonstrated by cell penetration through the Matrigel-coated polycarbonate filter in the Boyden chamber. The results showed that in Hela cell line, auraptene reduced cell invasion at concentration of $100 \mu \mathrm{M}$ (Fig.4A), while for A2780 cell line, inhibitory effect of auraptene on cell invasion was found at 25 and $50 \mu \mathrm{M}$, compared to control (Fig.4B).

\subsection{Effect of auraptene on MMP-2, MMP-9 en zymes activity}

Matrix metalloproteinases are important enzymes that degrade extracellular matrix (ECM) components and so play important roles tumor invasion and metastasis. In this regard, to investigate the inhibitory effects of auraptene on MMP-2 and MMP-9 enzyme activities, gelatin zymograph assay was applied. In our study, MMP-9 activity was significantly reduced at $12.5-100 \mu \mathrm{M}$ auraptene in Hela cell line. These results showed that reduction in MMP-2 enzymatic activity by auraptene was in a concentration-dependent manner (Fig.5A).

In A2780 cell line, MMP-2 and MMP-9 activity was significantly reduced at all examined concentrations compared to control. Maximum decrease occurred at $50 \mu \mathrm{M}$ auraptene. Reduction in enzymatic activity was in a concentration-dependent manner (Fig.5B).

Notably, the inhibitory effect of auraptene seems to be more on MMP-2 than MMP-9, at same concentrations of auraptene in both cell lines.

\section{Discussion}

Invasion and metastasis are exceedingly complex processes, and their genetic and biochemical determinants remain incompletely understood. Increased cell migration, reduction in cell adhesion, intracellular matrix disintegration and invasion to other organs are correlated with metastasis [26].

Matrix metalloproteinases are the best enzymes candidates for metastasis. Among MMPs, MMP-2 and MMP-9 have pivotal role in angiogenesis, tumor proliferation and invasion. Hence, they could be promising in developing chemotherapeutic drugs and preventing metastasis [27].

Cervical and ovarian cancers are the fourth and the fifth common cancers among women worldwide, respectively. Thus, there is an urgent need for major research to shed further light on these cancers [3].

Auraptene is a natural citrus coumarin found in many plants of Rutaceae, Apiaceae and Composite. However, the effect of auraptene on metastasis in cervical and ovarian cancers and on MMPs activity is still unknown. The aims 
of this study were to investigate the effect of auraptene on metastasis in cervical and ovarian cancers and MMP- 2 and MMP-9 activity, as well. In order to study of auraptene on cell viability, MTT test was done. Auraptene significantly reduced cell viability in both cell lines, compared to control and its inhibitory effect was in dose- dependent manner.

In another experiment, after 72 hours of incubation with increasing concentration of auraptene in three prostatic cell lines (LNCaP, DU145, PC3) cell viability was inhibited in dose-dependent manner [28]. Additionally, Zheng et al demonstrated the effect of auraptene at two different concentrations in colon cancer cell line. In this regard, auraptene inhibited cell viability, DNA cleavage induction and cell proliferation. The inhibitory effect of auraptene, was probably related to G1/S arrest and induction of apoptosis [29].

The effect of auraptene on cell migration was studied by wound healing assay. After 24 hours of incubation, there were no differences between control and auraptene in both cell lines and wound healing occurred.

In A2780 cell line, after 72 hours of incubation, wound healing occurred only in control group. It was revealed that all concentrations of auraptene have inhibitory effect on cell migration and it was dose-dependent.

Further investigation illustrated that in HUVEC cell line, auraptene inhibited cell migration and proliferation, compared to control [30]. Also, in a recent study, in oral epithelial cells, wound closure was improved by $100 \%$ in the presence of $>1 \mu \mathrm{M}$ auraptene [31].

In our study, in lower concentrations, auraptene needed more times to inhibit cell migration in A2780 cell line. In fact, cell migration ability in A2780 cell was lower than Hela cell line, and inhibitory effect of auraptene initiated after 48 hours. In Hela cell line, treatment with auraptene was significantly different from control group, after 6 hours of incubation.

In order to study of cell invasion, matrigel invasion assay was performed. In both cell lines, auraptene had dose dependent inhibition on cell migration and invasion.

In our study, gelatin zymography as complementary test was done to detect MMP-2 and MMP-9 activity. Significant change in MMP-2 and MMP-9 activity was represented in both cell lines after treatment with auraptene.

Recently, a study reported the effect of auraptene on MMP-2 and MMP-9 activity inducted by dextran sulfate sodium in tumor cells. In this study, auraptene inhibited pro-MMP-2 and pro-MMP-9 expression compared to control. While, dextran sulfate sodium increased MMP enzyme activity to amount of 13 fold for pro-MMP-2 and 31 fold for MMP-9 [21]. In fact, MMPs activation are through junction to urokinase-type plasminogen activator receptor (UPAR) and then plasminogen is converted to plasmin by uPA. Afterwards, plasmin converts pro-MMP to MMP. Therefore, the relationship between UPA and UPAR are an initial step in tumor invasion [27].

Increasing evidence implied that auraptene reduced the production of pro-MMP-7 protein without influence on mRNA expression in HT-29 cell line. This phenomenon was related to mTOR inhibition activity of auraptene. Furthermore, reduction of pro-MMP-7 translation was corre- lated with inhibition of 4EBP1 and elF4B phosphorylation which activated byERK1/2 pathway [30]. Further investigation showed that intraperitoneal injection of osthole as natural coumarin, reduced infarct volume and matrixmetalloproteinase- 9 activity after transient focal cerebral ischemia in rats [32].

Other studies on anticancer effect of some coumarins have been reported. Among terpenoid coumarins, examined in Raji cell line, including auraptene, umbelliprenin, badrakemone, methyl galbanate, galbanic acid, feselol, mogoltacin, conferone and ferukrinone, Galbanic acid and umbelliprenin were showed to have inhibitory effect on matrix metalloproteinase enzymes. Also, it was concluded that auraptene and umbelliprenin, would have precious anti-tumor-promoting effects in many carcinogenic cycles [33].

\section{Conclusion}

In conclusion, our findings indicated that auraptene efficiently suppresses the migration and invasion capacity of human cervical (Hela) and ovarian (A2780) cancer cells by reduction of MMP-2 and MMP-9 activity. Considering that there are some unreliable results for performed tests about high concentration $(100 \mu \mathrm{M})$ of auraptene, our data suggest that the possibility of auraptene as a potential anti-invasive and anti-migratory candidate should be further investigated in required concentrations in future studies to reveal their detailed mechanisms and functions.

\section{Acknowledgment}

This work was supported financially by a research grant (Grant No. 931687) from the Vice Chancellor for Research of Mashhad University of Medical Sciences, Mashhad, Iran. The results described in this paper were part of a PharmD student thesis.

\section{Conflict of interest}

The authors declared that there are no conflicts of interest.

\section{References}

1. Koh WJ, Greer BE, Abu-Rustum NR, Apte S M, Campos $\mathrm{SM}$, Cho KR, et al. Cervical cancer, version 2.2015. J Natl Compr Canc Netw. 2015;13(4):395-404.

2. Wu H, Wang S, Weng D, Xing H, Song X, Zhu T, et al. Reversal of the malignant phenotype of ovarian cancer A2780 cells through transfection with wild-type PTEN gene. Cancer Lett. 2008;271(2):205-214.

3. Roomi MW, Kalinovsky T, Rath M, Niedzwiecki A. A Specific Mixture of Nutrients Suppresses Ovarian Cancer A-2780 Tumor Incidence, Growth, and Metastasis to Lungs. Nutr. 2017;9(3):303.

4. Hiratsuka S, Nakamura K,Iwai S, Murakami M,Itoh T, Kijima H, etal.MMP9inductionbyvascularendothelialgrowthfactorreceptor-1isinvolvedinlung-specificme- 
tastasis.CancerCell.2002;2(4):289-300.

5. Sounni NE, Devy L, Hajitou A, Frankenne F, MunautC, Gilles C, et al. MT1-MMP expression promotes tumor growth and angiogenesis through an up-regulation of vascular endothelial growth factor expression. The FASEB J. 2002;16(6):555-564.

6. Clark IM, YoungDA,RowanAD. Matrix metalloproteinase protocols: Springer; 2001.

7. Luca M, HuangS,GershenwaldJE, Singh RK, Reich R, Bar-Eli M. Expression of interleukin-8 by human melanoma cells up-regulates MMP-2 activity and increases tumor growth and metastasis. Am J Pathol. 1997;151(4):1105-11013.

8. MannelloF, Gazzanelli G. Tissue inhibitors of metalloproteinases and programmed cell death: conundrums, controversies and potential implications. Apoptosis. 2001;6:479-482.

9. Sang Q-XA, Jin Y,Newcomer RG,Monroe SC,Fang X, Hurst DR, etal.Matrixmetalloproteinaseinhibitorsasprospectiveagentsforthepreventionandtreatmentofcardiovascularandneoplasticdiseases.CurTopMedChem.2006;6(6):289-316.

10. Genovese S, Epifano F. Auraptene: a natural biologically active compound with multiple targets. Cur Drug Targets. 2011;12(3):381-386.

11. Curini M, Cravotto G, Epifani F, Giannone G. Chemistry and biological activity of natural and synthetic prenyloxycoumarins. Curr Med Chem. 2006;13(2):199-222.

12. Okuyama S, Minami S, Shimada N, Makihata N, Nakajima M, Furukawa Y. Anti-inflammatory and neuroprotective effects of auraptene, a citrus coumarin, following cerebral global ischemia in mice. Euro J Pharmacol. 2013;699(1-3):118-123.

13. Prince M, Li Y, Childer A, Itoh K, Yamamoto M, Kleiner HE. Comparison of citrus coumarins on carcinogen-detoxifying enzymes in Nrf2 knockout mice. Toxicol Lett. 2009;185(3):180-186.

14. Wu L, Wang X, Xu W, Farzaneh F, Xu R. The structure and pharmacological functions of coumarins and their derivatives. Curr Med Chem. 2009;16(32):4236-4260.

15. Takeda K, Utsunomiya H, Kakiuchi S, Okuno Y, Oda k, Inada KI, et al. Citrus auraptene reduces Helicobacter pylori colonization of glandular stomach lesions in Mongolian gerbils. J Oleo Sci. 2007;56(5):253-260.

16. Furukawa Y, Watanabe S, Okuyama S, Nakajima M. Neurotrophic effect of citrus auraptene: neuritogenic activity in PC12 cells. Int J Mol Sci. 2012;13(5):53385347.

17. Tanaka T, Sugiura H, Inaba R, Nishikawa A, Murakami A, Koshimizu K, et al. Immunomodulatory action of citrus auraptene on macrophage functions and cytokine production of lymphocytes in female BALB/c mice. Carcinog.1999;20(8):1471-1476.

18. Soltani F, Mosaffa F, Iranshahi M, Karimi G, Malekaneh M, Haghighi F. Auraptene from Ferula szowitsiana protects human peripheral lymphocytes against oxidative stress. Phy Res. 2010;24(1):85-89.

19. Krishnan P, Yan KJ, Windler D, Tubbs J, Grand R, Li BDL, et al. Citrus auraptene suppresses cyclin D1 and significantly delays $\mathrm{N}$-methylnitrosourea induced mammary carcinogenesis in female Sprague-Dawley rats. BMC Cancer. 2009;9:259.

20. Tanaka T, Kawabata K, Kakumoto M, Hara A, Murakami A, Kuki W, et al. Citrus auraptene exerts dose-dependent chemopreventive activity in rat large bowel tumorigenesis: the inhibition correlates with suppression of cell proliferation and lipid peroxidation and with induction of phase II drug- metabolizing enzymes. Cancer Res. 1998;58(12):2550-2556.

21. Kawabata K, Murakami A, Ohigashi H. Auraptene decreases the activity of matrix metalloproteinases in dextran sulfate sodium-induced ulcerative colitis in ICR mice. Biosci Biotechnol Biochem. 2006;70(12):30623065.

22. Rodriguez LG, Wu X, Guan JL. Wound-healing assay. Cell Migration: Developmental Methods and Protocols. 2005;23-29.

23. Moreno-Bueno G, PeinadoH,MolinaP,OlmedaD,CubilloE,SantosV,etal. The morphological and molecular features of the epithelial-to-mesenchymal transition. Nat Protoc. 2009;4(11):1591-1613.

24. Keenan TM, Folch A. Biomolecular gradients in cell culture systems. Lab Chip. 2008;8(1):34-57.

25. Frankowski H, Gu YH, HeoJH,MilnerR,DelZoppo GJD. Use of gel zymography to examine matrix metalloproteinase (gelatinase) expression in brain tissue or in primary glial cultures. Astrocytes: Methods and Protocols. 2012;221-33.

26. Hanahan D, Weinberg RA. The hallmarks of cancer. Cell. 2000;100(1):57-70.

27. Chen HJ, Lin CY, Lee CY, Shih NC, Peng SF, Tsuziki M, et al. Kaempferol suppresses cell metastasis via inhibition of the ERK-p38-JNK and AP-1 signaling pathways in U-2 OS human osteosarcoma cells. Oncol Rep. 2013;30(2):925-32.

28. Tang MX, Ogawa K, Asamoto M, Hokaiwado N, Seeni A, Suzuki A, et al. Protective effects of citrus nobiletin and auraptene in transgenic rats developing adenocarcinoma of the prostate (TRAP) and human prostate carcinoma cells. Cancer Sci. 2007;98(4):471-7.

29. Zheng Q, Hirose Y, Yoshimi N, Murakami A, Koshimizu K, Ohigashi H, et al. Further investigation of the modifying effect of various chemopreventive agents on apoptosis and cell proliferation in human colon cancer cells. J Cancer Res Clin Oncol. 2002;128(10):539-46.

30. Wang S, Yoon YC, Sung MJ, Hwang JT, Hur HJ, Kim HJ, et al. Citrus-derived auraptene stimulates angiogenesis by activating the Erk-and PI3K/Akt/eNOS-dependent signaling pathways in human umbilical vein endothelial cells. J Funct Foods. 2012;4(4):899-905.

31. La VD, Zhao L, Epifano F, Genovese S, Grenier D. Anti-inflammatory and wound healing potential of Citrus auraptene. J Med Food. 2013;16(10):961-4.

32. Mao X, Yin W, Liu M, Ye M, Liu P, Liu J, et al. Osthole, a natural coumarin, improves neurobehavioral functions and reduces infarct volume and matrix metalloproteinase-9 activity after transient focal cerebral ischemia in rats. Brain Res. 2011;1385:275-80.

33. Iranshahi M, Kalategi F, Rezaee R, Shahverdi AR, Ito C, Furukawa $\mathrm{H}$, et al. Cancer chemopreventive activity of terpenoid coumarins from Ferula species. Planta medica. 2008;74:147-150. 


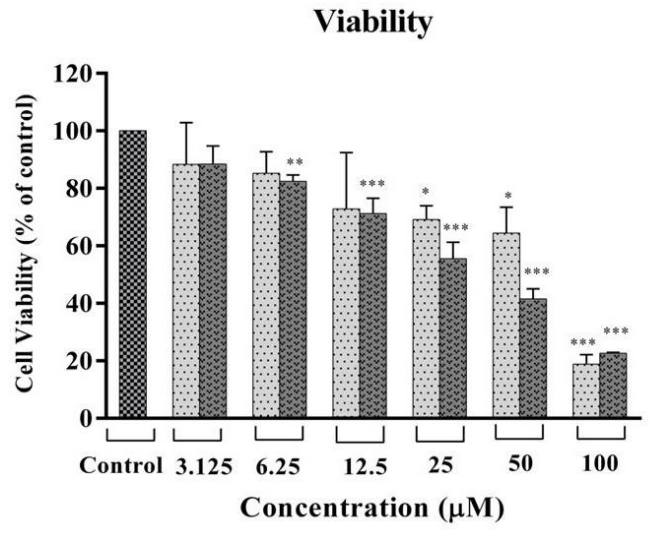

Figure 1 The effects of auraptene on the viability of Hela and A2780 cell lines. Cells were exposed to various concentrations of auraptene at $37^{\circ} \mathrm{C} .(\mathrm{n}=3)$
A2780

Hela
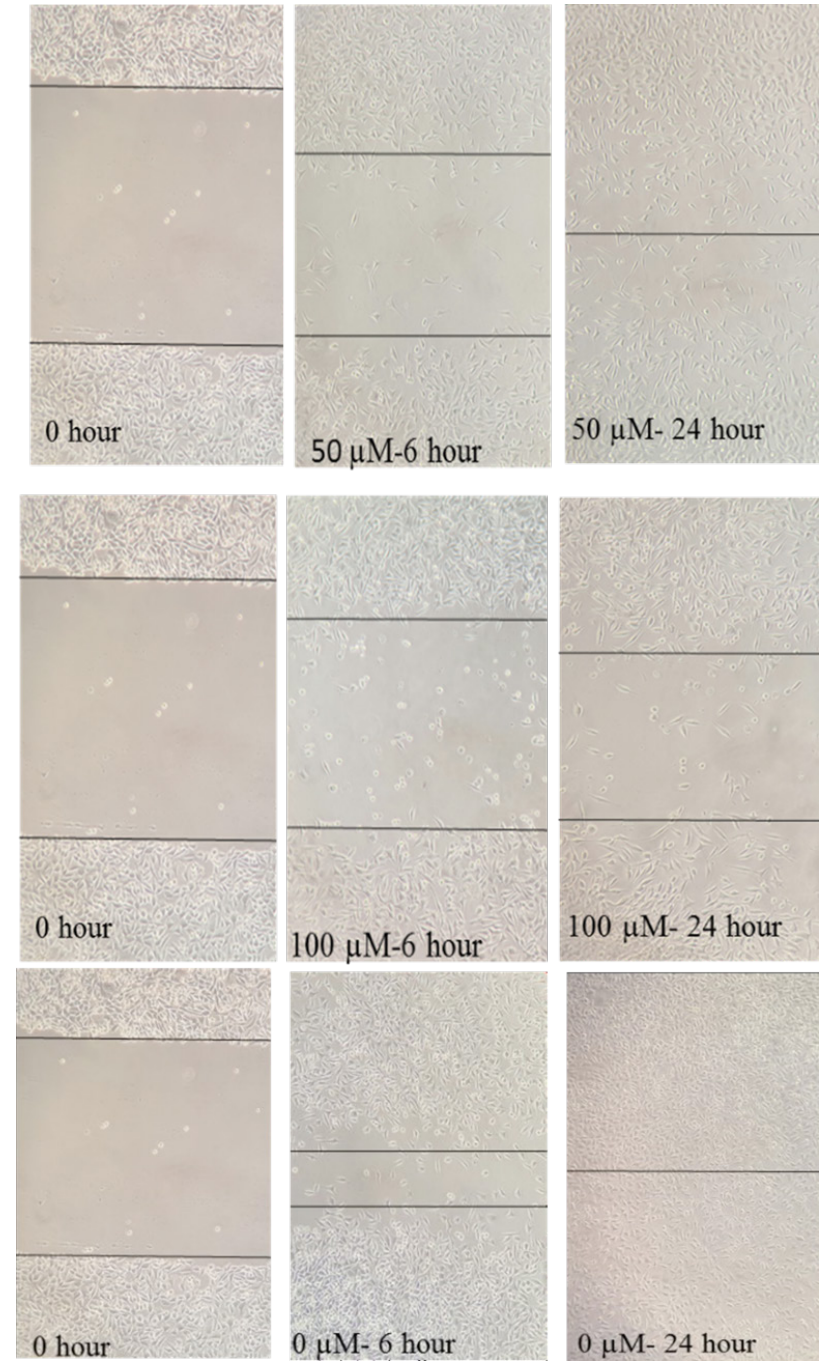

Figure 2A Effect of aurapfetene on Hela cell migration. Cell migration was significantly reduced by auraptene at $100 \mu \mathrm{M} .(\mathrm{n}=4)$

\section{Migration-Hela}

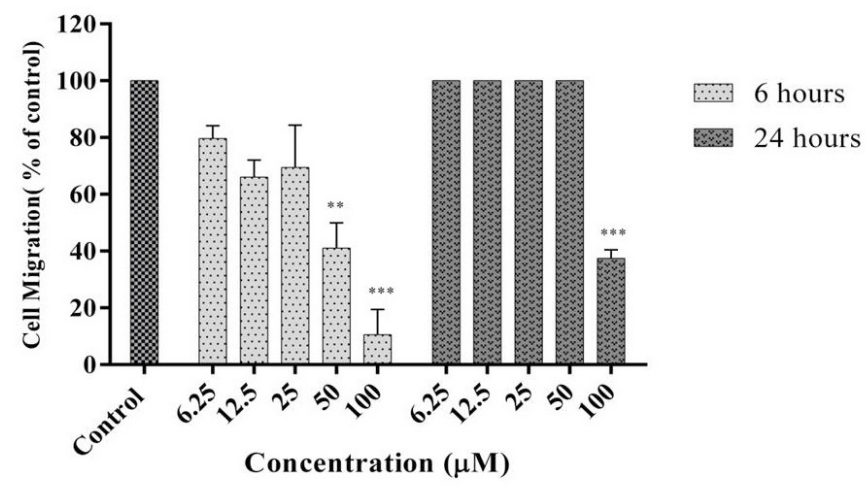

Figure 2B Effect of auraptene on cell migration in a time dependent manner. $(n=4)$ 

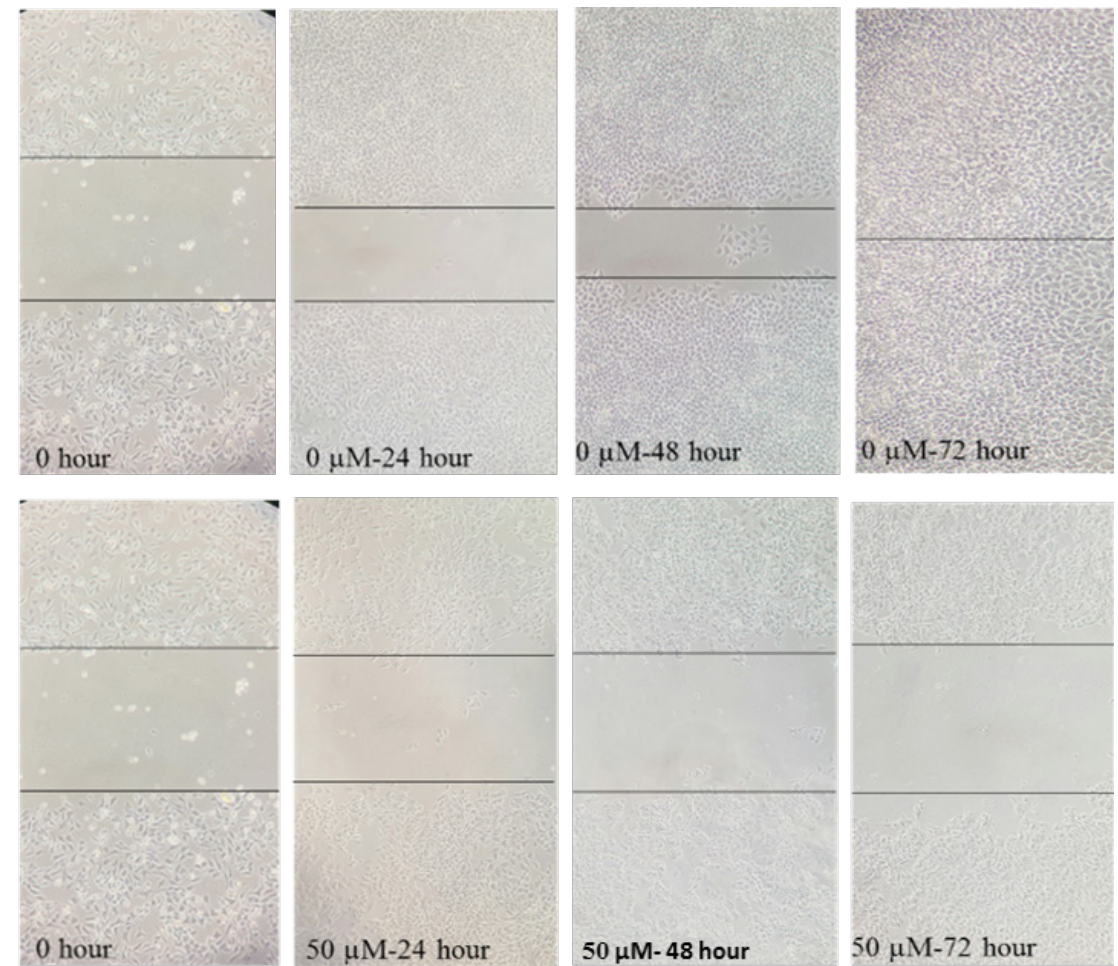

$50 \mu \mathrm{M}-24$ hour

$50 \mu \mathrm{M}-72$ hour

Figure 3A Effect of auraptene on A2780 cell migration. Cell migration was significantly reduced by auraptene at $50 \mu \mathrm{M}$. $(\mathrm{n}=4)$

Migration-A2780

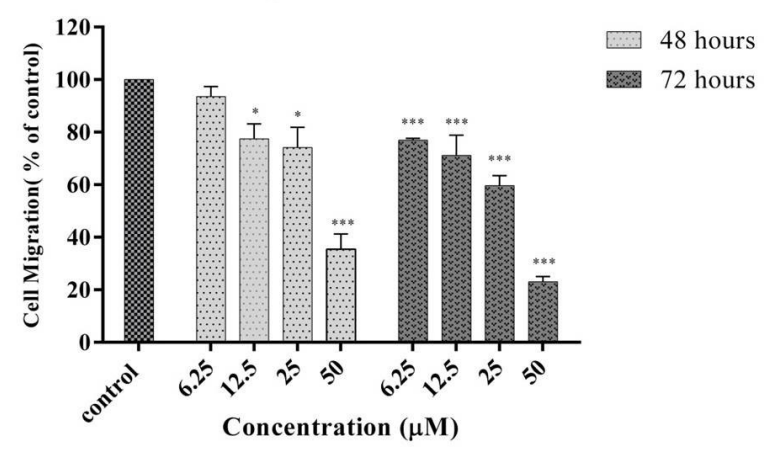

Figure 3B Effect of auraptene on cell migration in a time dependent manner. $(\mathrm{n}=4)$ 
Hela-Matrigel

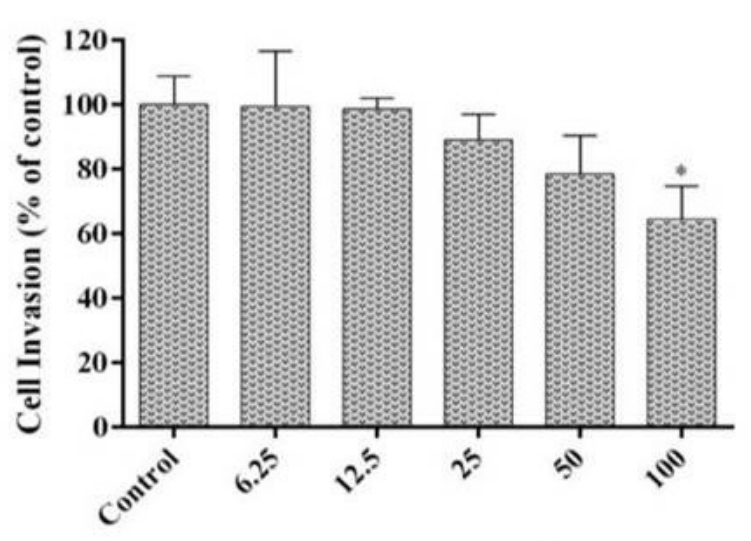

Concentration $(\mu \mathrm{M})$

Figure 4A Inhibitory effect of auraptene on Hela cell invasion, after 24 hours incubation. $(n=2)$

Hela-MMP2 $\backslash 9$

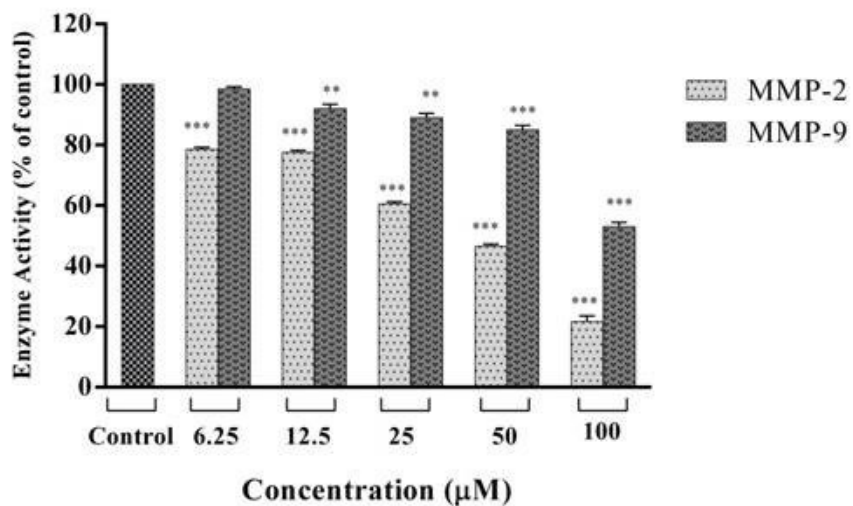

Figure 5A Effect of different concentrations of auraptene on MMP-2 and -9 activity in Hela cells. $(\mathrm{n}=2)$
A2780-Matrigel

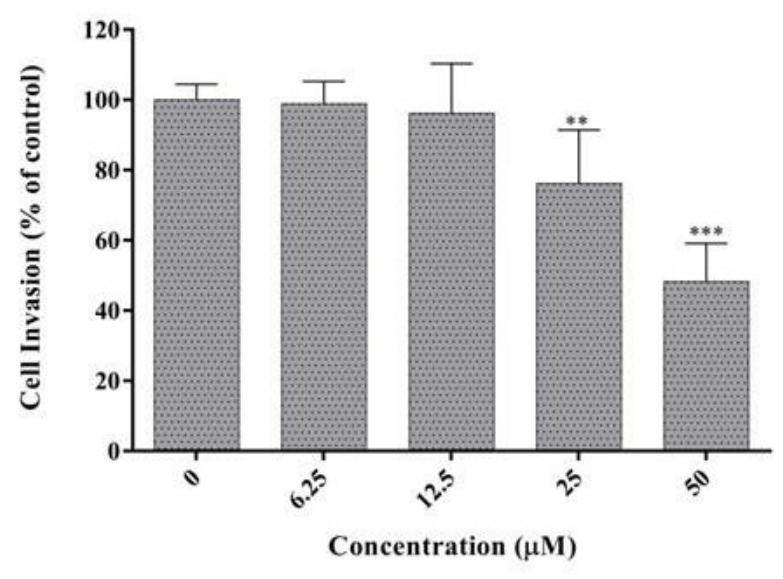

Figure 4B Inhibitory effect of auraptene on A2780 cell invasion, after 24 hours incubation. $(\mathrm{n}=2)$

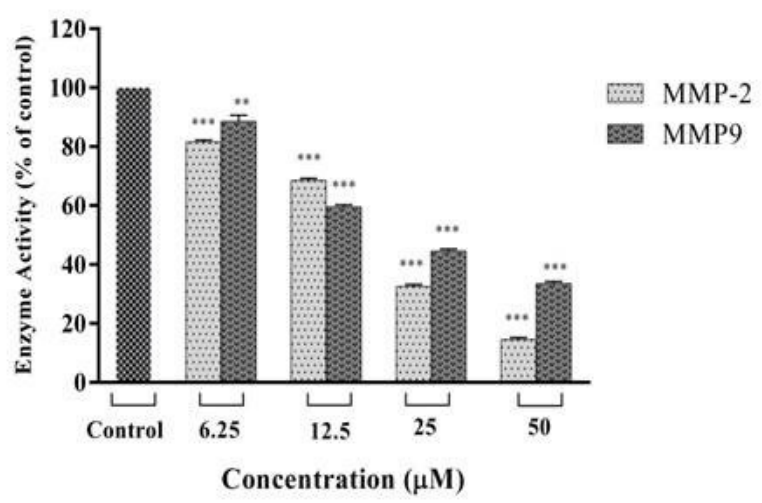

Figure 5B Effect of different concentrations of auraptene on MMP-2 and -9 activity in A2780 cells. ( $\mathrm{n}=2$ ) 